\title{
La pratique narratologique
}

The Narratological Praxis

\section{Arnaud Schmitt}

\section{OpenEdition}

\section{Journals}

Édition électronique

URL : https://journals.openedition.org/questionsdecommunication/11117

DOI : 10.4000/questionsdecommunication. 11117

ISSN : 2259-8901

\section{Éditeur}

Presses universitaires de Lorraine

\section{Édition imprimée}

Date de publication : 1 septembre 2017

Pagination : 215-229

ISBN : 9782814303256

ISSN : 1633-5961

\section{Référence électronique}

Arnaud Schmitt, «La pratique narratologique », Questions de communication [En ligne], 31 | 2017, mis en ligne le 01 septembre 2019, consulté le 26 octobre 2021. URL : http://journals.openedition.org/ questionsdecommunication/11117 ; DOI : https://doi.org/10.4000/questionsdecommunication. 11117 


\title{
$>$ ÉCHANGES
}

\author{
ARNAUD SCHMITT \\ Cultures et littératures des mondes anglophones \\ Université de Bordeaux \\ $\mathrm{F}-33000$ \\ arnaud.schmitt@u-bordeaux.fr
}

\section{LA PRATIQUE NARRATOLOGIQUE}

\begin{abstract}
Résumé. - Je reviens sur trois aspects développés par Raphaël Baroni dans « L'Empire de la narratologie, ses défis et ses faiblesses » : l'histoire de cette discipline, ses frontières et sa place à l'université (principalement française). Dans un premier temps, je m'efforce de démontrer que plus que géographique, sa logique historique est bipolaire ; selon moi, la narratologie est définie par les deux pôles qui déterminent sa cohérence disciplinaire depuis plusieurs décennies : elle oscille en effet, selon l'approche que l'on revendique, entre le formalisme et la théorie de la réception. J'avance ensuite que ce pôle appelé par les américains « reader-oriented » est celui qui a mené la narratologie à élargir ses frontières afin d'intégrer des outils d'analyse provenant d'autres champs, telles que les sciences cognitives. Néanmoins, afin de ne pas perdre sa cohérence disciplinaire, la narratologie ne doit pas perdre de vue la praxis qui la sous-tend, l'analyse étroite du texte. Enfin, je démontre que, pour diverses raisons structurelles, la place de la narratologie française à I'Université n'a jamais été aussi fragile, et reste menacée par la « logique géographique » qui structure les études littéraires en France.
\end{abstract}

Mots clés. - narratologie, théorie littéraire, formalisme, théorie de la réception, enseignement de la narratologie 
$\mathrm{D}$ ans « L'empire de la narratologie, ses défis et ses faiblesses », Raphaël Baroni (2016) dresse un tableau à la fois précis et complet, véritable gageure, de l'état des études narratologiques aujourd'hui. Sa profonde connaissance du sujet lui permet de saisir de manière concise l'évolution historique d'une discipline qui n'a pas toujours été perçue comme telle, et d'en présenter les principaux enjeux. J'en retiens cinq : un certain déclin de la narratologie à l'aube du $X X l^{e}$ siècle, après l'âge d'or des années 70 ; une évolution entropique des études narratives - qui dépassent largement le cadre de la narratologie - due en grande partie à l'ubiquité du récit comme objet d'étude pluridisciplinaire (« l'empire du récit ») ; les indéniables implications éthiques découlant de cette entropie ; enfin, la nécessité pour la narratologie d'exister en tant que lieu institutionnel, discipline à part entière, et non simple « boîte à outils ».

Faire l'économie d'une réflexion sur ces cinq aspects mis au jour par Raphaël Baroni reviendrait selon moi à sonner, à plus ou moins long terme, le glas de la narratologie ; ou en tout cas à la cantonner à ce qu'elle est d'ailleurs peu ou prou devenue : un champ de recherche restreint (non pas de par son amplitude scientifique, mais, tout simplement, du fait du nombre très réduit de « narratologues pratiquants »). En effet, son analyse est d'autant plus précieuse qu'elle arrive à un moment où la théorie du récit, les études narratives ou encore la poétique cognitive semblent reléguer la narratologie, et le formalisme qui lui est forcément intrinsèque, au rang des disciplines poussiéreuses et caduques.

Plutôt que de contester le constat effectué dans son article, constat que je partage en grande partie, je souhaiterais développer trois des cinq points énoncés ci-dessus : la dimension historique de la narratologie, l'évolution de ses frontières et son identité institutionnelle.

\section{La taille de l'empire et son histoire}

L'affirmation de Raphaël Baroni selon laquelle la narratologie « a connu son heure de gloire durant le "moment structuraliste" autour des années 1965-1975 » (ibid. : 220) est irréfutable mais peut néanmoins être accompagnée de quelques précisions. On peut dans un premier temps s'accorder sur le fait que cette observation s'applique avant tout à la France. Outre-Atlantique, la narratologie s'est développée autour des mêmes dates - le texte fondateur de la narratologie américaine, The Rhetoric of Fiction de Wayne C. Booth, est publié en 196I - mais selon une dynamique différente, dont le sort n'a pas forcément été lié à celui du structuralisme!. II est assez difficile de définir avec précision la chronologie

\footnotetext{
' La vague déconstructionniste déferlant surYale, puis sur certaines grandes universités américaines, autour des années 70 et 80, et la création du label French Theory - désignant à la fois des philosophes, mais aussi des théoriciens de la littérature - ont entrainé selon moi une différence sensible entre la perception de la narratologie sur le continent nord-américain et en Europe, ou en tout cas un brouillage des frontières.
} 
d'un mouvement théorique tel que la narratologie, et l'on se heurte rapidement aux écueils habituels, par exemple, la façon de mesurer sa courbe d'intensité : se mesure-t-elle selon une logique qualitative (la publication des grands textes qui ont défini les contours de la discipline) ou quantitative (le nombre de textes relevant de la discipline et publiés durant une période définie $\left.{ }^{2}\right)$ ? Et le problème corollaire : comment avoir accès à des telles données ? Comme précisé ci-dessus, lorsque Raphäl Baroni situe le sommet de cette courbe autour des années 1965-1975, cela semble relever de l'évidence, mais on se trouve alors probablement dans une logique qualitative (premiers textes importants sur le sujet de Tzvetan Todorov, Gérard Genette, Roland Barthes...).

Un autre écueil concerne la difficulté de procéder à une étude comparative lorsqu'il s'agit du développement de la narratologie d'un pays à un autre, notamment parce que la définition du champ de recherche peut varier, mais aussi du fait de politiques universitaires et éditoriales différentes. Ne négligeons pas non plus l'influence de l'objet étudié sur les outils narratologiques utilisés ${ }^{3}$, il est commun de faire remarquer que la narratologie aurait été probablement différente si Gérard Genette avait eu un autre idéal littéraire que l'œuvre de Marcel Proust. Les modèles narratifs inhérents à la littérature américaine sont distincts de ceux que l'on retrouve dans la littérature française, même si, bien évidemment, des similarités existent. Or, les textes déterminent les moyens mis en place pour les observer, et cela est forcément souhaitable. Sans avoir de données précises pour étayer mes propos, je peux néanmoins avancer que la courbe d'intensité de l'évolution de la narratologie aux États-Unis n'atteint pas les mêmes sommets qu'en France, mais elle est plus constante, plus stable. En d'autres termes, la diffusion de la narratologie outreAtlantique s'inscrit dans une logique soutenue, mais caractérisée par une intensité plus basse me semble-t-il, et s'effectue par le biais de quelques entités universitaires, les universités de Chicago et de l'Ohio, pour ne citer qu'elles, l'Issn (International Society for the Study of Narrative, association qui a beau être « internationale », mais dont l'identité demeure fortement américaine) ou la revue Narrative. De plus, bien qu'elle ne perde jamais de vue les évolutions narratologiques européennes (les concepts de Gérard Genette ont été discutés par la plupart des grands narratologues américains, notamment par James Phelan), la narratologie américaine, du fait de la nature de son texte fondateur et donc de par sa proximité avec d'autres théories littéraires, a très vite quitté le giron du structuralisme :

« La clé de voûte de cette approche est la conception que le récit est avant tout et essentiellement un acte communicatif. Dans les premiers stades de son développement, c'est-à-dire dans les années soixante et au début des années soixante-dix, cette approche recoupait de façon évidente à la fois l'ouvrage influent de Wayne Booth Rhetoric of Fiction (196I) et ce qu'on a appelé les "théories de la réception" » (Segal, 2015 : s.p.).

\footnotetext{
2 Un autre obstacle surgit alors : l'équivocité disciplinaire de certains textes théoriques.

3 «Discours du récit, qui prend comme seul domaine d'application l'œuvre de Proust, constitue une tentative pour bâtir ce que Genette appelle lui-même une "technologie du discours narratif" » (O'Kelly, 2012 : s.p.).
} 
II n'est ainsi pas surprenant que James Phelan ait défini sa propre approche de la narratologie comme essentiellement rhétorique. En effet, il revendique clairement l'héritage de Wayne $\mathrm{C}$. Booth et établit un lien direct entre l'approche narratologique de ce dernier et la sienne, présentée ainsi comme narratologie rhétorique : «Considérer le récit comme ayant pour finalité de communiquer des connaissances, des sentiments, des valeurs et des croyances équivaut à considérer le récit comme de la rhétorique $\gg^{4}$ (Phelan, 1996: 18, ma traduction - MT). || est tout à fait probable que Gérard Genette lui-même puisse partager cette vision et c'est paradoxalement dans son diptyque ${ }^{5}$ sur l'art qu'il s'en approche le plus -, mais, en majorité, ses propres études narratologiques, formelles et diachroniques (histoire des faits narratologiques pour être précis), ne le reflètent pas. De plus, un texte aussi essentiel dans le paysage narratologique américain que Before Reading de Peter J. Rabinowitz (1987) relève-t-il réellement de la narratologie selon une approche genettienne? Probablement pas, il renvoie bien plus aux travaux de l'École de Constance. Ce qu'on appelle couramment reader-oriented narratology s'apparenterait bien plus pour un narratologue français à la théorie de la réception qu'à l'étude formelle des procédés narratologiques d'un texte. La narratologie américaine que l'on peut ainsi différencier de - et non opposer à - la narratologie structuraliste ${ }^{6}$ et l'attention qu'elle porte à la réception est probablement l'un des facteurs qui ont mené à l'émergence de la poétique cognitive : à trop se poser la question de la lecture et de son impact sur le texte, on finit par parler de cognition. Nous reviendrons sur ce point essentiel un peu plus loin.

II n'y a donc a priori pas une narratologie mais bien des narratologies, ou en tout cas plusieurs approches, ce qui ne rend pas aisée la tâche de celui qui veut en synthétiser l'histoire. Certains ont d'ailleurs récemment remis en cause une dichotomie historique pourtant actée par grand nombre de praticiens de la discipline :

« Nous pensons que le problème qui se pose avec la distinction classique/post-classique peut difficilement être résolu en affinant la dichotomie de quelque manière que ce soit, et qu'il y a des limites à sa cohérence et ses applications théoriques. En effet, les caractéristiques qui déterminent la différence entre le classique et le post-classique sont aussi révélatrices des problèmes que pose une telle distinction : (I) une certaine inconsistance définitoire des termes (2); un manque de perspicacité théorique $[\ldots]^{7} \gg$ (Passalacqua, Pianzola, $2016: 196$, MT).

${ }^{4}$ «Viewing narrative as having the purpose of communicating knowledge, feelings, values, and beliefs is viewing narrative as metoric ».

${ }^{5}$ L'Furre de l'art: immanence et transcendance (1994) et L'Fuvre de l'art : la relation esthétique (1997).

${ }^{6}$ || me paraît préférable de définir cette seconde tendance selon son approche formaliste, et non en suivant une logique géographique car la narratologie européenne me semble moins homogène dans son approche que sa consœur américaine.

${ }^{7}$ « We think that the problems that arise with the classical/postclassical distinction can hardly be resolved by refining the dichotomy in any particular way and that there are limits to its theoretical soundness and applications. Indeed, the features that qualify the difference between classical and postclassical are also markers of the problems encountered by such a distinction: ( I) definitional looseness of the terms; (2) lack of theoretical insight $\gg$. 
De manière plus empirique, je me suis moi-même rendu compte, notamment lors de colloques, que ces deux termes renvoyaient à des champs de la narratologie différents d'un interlocuteur à un autre. Cependant, contrairement à ce que Franco Passalacqua et Federico Pianzola laissent entendre, la définition de cette dichotomie n'est pas si inconsistante ; elle oscille avant tout entre deux tendances : une définition chronologique (la narratologie structuraliste classique et la narratologie à partir des années 80 , sous toutes ses formes) ou méthodologique (la narratologie structuraliste et la narratologie qui se collète avec la réception, pour schématiser). Mais comme nous l'avons vu, Wayne C. Booth était déjà d'une certaine manière post-classique, alors que le classicisme en narratologie n'existait pas encore. Jan Alber et Monika Fludernik (20l0 : 2, MT) présentent un argument similaire dans l'introduction de Postclassical Narratology, ouvrage qu'ils ont dirigé : «Cependant, on pourrait avancer que ces représentants de la narratologie classique [Roland Barthes, Gérard Genette, Claude Bremond, Tzvetan Todorov, ou encore Algirdas J. Greimas, mais aussi Gerald Prince ou Seymour Chatman] avaient déjà commencé à s'éloigner du modèle structuraliste, même légèrement ou de manière à peine perceptible ${ }^{8} \gg$. Revenant sur le concept de David Herman, ils avancent néanmoins que la narratologie post-classique se distingue de quatre manières de la narratologie classique, affirmant ainsi le bien-fondé de la distinction : I) elle désigne les textes qui se penchent sur les éventuelles failles du paradigme originel ; 2) elle propose des extensions méthodologiques du modèle classique (linguistiques, psychanalystes...) ; 3) des extensions thématiques (féministes, postcoloniales...) ; 4) ou enfin des extensions médiatiques (cinéma, roman graphique...) (ibid. : 3). Réelles, ces quatre directions posent cependant une question qui me semble évidente : pourquoi ces catégories ne peuvent-elles pas être définies simplement par le terme narratologie ? Certaines proposent ou appliquent d'ailleurs des modèles d'analyse ouvertement structuralistes, et lorsqu'elles ne le font pas, elles semblent parfois bien éloignées des préoccupations de ce qu'on entend communément par narratologie, classique ou même post-classique. Jan Alber et Monika Fludernik présentent des arguments convaincants, mais en introduction d'un ouvrage qui, comme son nom l'indique, a intérêt à souligner la différence entre ces deux temps, ou paradigmes plus exactement, de la narratologie. Cependant, encore une fois, rien n'empêche de considérer ces évolutions de la narratologie comme intégrées. Je ne tiens pas à revenir plus longtemps sur cette dichotomie, elle a été maintes fois commentée, plutôt sur ce dont elle est le symptôme : une incertitude définitoire, entraînant une certaine inquiétude quant à la capacité de la narratologie à absorber d'autres disciplines sans perdre son identité, ou en tout cas une certaine cohérence disciplinaire. Avant de faire cela, je souhaite revenir une dernière fois sur l'histoire de la narratologie, et indirectement, sur sa définition. Comme j'ai pu le préciser, l'histoire de la discipline qui nous intéresse ici ne peut se contenter d'être déterminée de manière géographique (même si une logique géographique existe, comme j'ai pu le souligner) ou en termes de classicisme et

\footnotetext{
${ }^{8}$ «Yet, one could argue that these representatives of classical narratology already started to drift away from the structuralist model, if ever so slightly and imperceptibly ».
} 
post-classicisme ; en revanche, elle peut être représentée par un axe disciplinaire qui s'étend entre deux pôles que seraient la narratologie structuraliste ou formaliste et la reader-oriented narratology (que je rechigne, au vu de son évolution, à cantonner au continent américain car un chercheur comme Raphäl Baroni me semble parfaitement correspondre à cette tendance). Ces deux pôles ne s'opposent pas. Bien au contraire. Ils définissent et délimitent selon une logique dialectique ce qu'une communauté de chercheurs comprend par narratologie, permettant à la fois une diversité d'approches tout en conservant une cohérence paradigmatique. Hélas, ces pôles ont parfois du mal à contenir des initiatives théoriques innovantes et très prometteuses, mais qui remettent en cause selon moi l'idée de narratologie.

\section{Les frontières de la narratologie}

La narratologie peut-elle être cognitive ? Une telle catégorie existe-t-elle ? Peut-elle même rejoindre le champ de la neuro-esthétique? Le fait que je puisse poser ces questions - légitimes puisque le concept de narratologie cognitive, comme le révèle une brève recherche sur l'internet, apparaît dans un nombre élevé de publications - est révélateur de l'existence des nombreuses extensions méthodologiques qui, selon Jan Alber et Monika Fludernik, caractérisent la narratologie post-classique ; cette narratologie-là, si elle est encore narratologique, est en tout cas bien souvent post-formaliste. Cette impression de dissémination semble partagée par Jan C. Meister (20 I3, MT) qui, dans sa définition de la narratologie pour le Living Handbook of Narratology, insiste autant sur les extensions thématiques que sur les extensions méthodologiques, l'évolution historique de la narratologie étant avant tout caractérisée, d'après lui, par son étude de schémas narratifs de plus en plus variés, et surtout extérieurs à la littérature :

« Dominée par les approches structuralistes à ses débuts, la narratologie a donné lieu à une variété de théories, concepts et procédures analytiques. Ses concepts et ses modèles sont largement utilisés comme outils heuristiques, et les théorèmes narratologiques jouent un rôle central dans l'exploration et la modélisation de notre capacité à produire et comprendre des récits à travers une multitude de formes, médias, contextes et pratiques communicationnelles ${ }^{9} \gg$.

Le terme de dissémination utilisé plus haut contient forcément un aspect négatif, ou en tout cas implique un certain glissement des frontières méthodologiques et thématiques de la narratologie telle qu'elle fut envisagée par ses fondateurs, mais on ne retrouve pas cette réticence dans la citation de Jan C. Meister. Bien que forcément brève, sa définition souligne plutôt la polyvalence de l'outil narratologique et ce qui pourrait être à la fois sa force principale et son salut : sa faculté à pouvoir être utilisée ailleurs (un ailleurs méthodologie ou thématique donc). C'est cet ailleurs qui peut

\footnotetext{
9 « Dominated by structuralist approaches at its beginning, narratology has developed into a variety of theories, concepts, and analytic procedures. Its concepts and models are widely used as heuristic tools, and narratological theorems play a central role in the exploration and modeling of our ability to produce and process narratives in a multitude of forms, media, contexts, and communicative practices ».
} 
poser problème, ou plus exactement le fait que pour être exportable, la narratologie doive se résigner à n'être qu'une « boîte à outils », aspect problématique comme le souligne Raphaël Baroni (2016 :227). II est difficile d'affirmer de manière impérieuse que la mutation de la narratologie de discipline à part entière en une palette d'outils prêts à être utilisés par d'autres disciplines est une bonne ou une mauvaise chose. Rien ne dit d'ailleurs qu'elle ne puisse être les deux à la fois, ce qui semble être le cas pour l'instant ; cela lui permettrait d'accepter que certains de ses outils soient quelque peu « dénaturés », puisqu'elle conserverait néanmoins ce qu'on pourrait appeler un noyau disciplinaire historique. Cependant, rien n'indique non plus que cette « exportabilité » ne finisse pas par se retourner contre elle et, en parallèle d'un éparpillement méthodologique ou thématique, sonner le glas du concept unifié de narratologie (qui subsiste pour l'instant malgré sa scission en deux périodes historiques, évoquées plus haut) pour ne laisser que quelques outils narratologiques. C'est une menace concrète. Une autre existe, sûrement plus importante encore, et elle est à nouveau soulignée par Raphaël Baroni : la toute-puissance du tournant narratif. Ce qui pose le problème inverse : non pas la propension de la narratologie à exporter ses outils, mais bien à en importer d'autres.

Le récit est maintenant partout, ou il l'a été pendant ces vingt dernières années. En effet, son influence est maintenant quelque peu relativisée, du moins par certains théoriciens. Raphaël Baroni cite comme exemple le fameux article de Galen Strawson (2004 : 430), « Against Narrativity », dans lequel ce dernier établit une distinction entre une structure ontologique narrative et épisodique, se réclamant luimême de la seconde tendance et avouant ne pas se retrouver dans le tout narratif. Adoptant une logique différente, mais parvenant à des conclusions similaires, Paul Bloom (2012) exprime à son tour un certain scepticisme quant au prétendu rôle central joué par le narratif dans nos constructions identitaires et nos actes cognitifs quotidiens. Reprenant à son compte certaines critiques émises par Jonathan Kramnick (20l I) (tout en critiquant certaines failles dans son raisonnement) à l'encontre de ce que ce dernier a appelé le « darwinisme littéraire »- une version du tout narratif qui veut que le récit et, plus généralement le littéraire, fasse partie intégrante de notre structure métaphysique - il insiste sur le fait qu'accorder une telle place au récit ou au narratif dans l'évolution de la pensée humaine ne repose sur aucune fondation scientifique solide, en tout cas pas dans sa version la plus extrême, à savoir une forme d'ubiquité proclamée du narratif (ibid. : 391). De manière tout à fait similaire, Steven Pinker (1994 : 57-58) avait fustigé le tournant linguistique, et la soudaine souveraineté du langage devenu base cognitive indéboulonnable de tout acte de connaissance ; et ce, en rétablissant la différence fondamentale entre pensée et langage. Le darwinisme littéraire mentionné ci-dessus témoigne d'une démarche réductionniste identique. Dans un article essentiel, « Narratologie et sciences cognitives : une relation problématique », Marie-Laure Ryan (2015) revient sur ce type de démarches réductionnistes et fait le bilan de trois décennies durant lesquelles le champ littéraire a largement investi des domaines scientifiques qui lui étaient jusque-là totalement étrangers. Elle revient aussi sur cette volonté de voir du récit partout, volonté qu'elle nomme « inflationniste »: 
«L'une des caractéristiques des théories inflationnistes est qu'elles traitent le récit comme un tout sans distinguer des constituants spécifiques. Selon ces théories, ce serait la possession innée d'une faculté narrative, faculté comparable à l'idée que Chomsky se fait de la compétence linguistique et de la grammaire universelle, qui nous permettrait d'avoir un sens de notre identité, d'acquérir une théorie de l'esprit, de former des souvenirs, ou de saisir le vécu de l'expérience personnelle » (ibid. : I2).

Mais Marie-Laure Ryan ne se limite pas l'inflationnisme narratif, elle met en évidence les limites certaines de ce que communément nous appelons poétique cognitive, en insistant sur une certaine naïveté des sciences humaines face aux données scientifiques et leur interprétation, pour résumer ses propos brièvement, mais aussi sur notre incapacité à évaluer la validité des conclusions scientifiques que nous utilisons ensuite dans nos publications. Elle parle ensuite plus spécifiquement de la narratologie cognitive, sujet qui nous intéresse au premier chef dans le cadre de cet article, qu'elle juge tout aussi sévèrement:

«Mais cetemprunt de concepts scientifiques neva pasjusqu'à l'adoption des rigueurs de l'expérimentation. Les travaux qui passent comme exemples de narratologie cognitive restent strictement spéculatifs, et certains narratologues fascinés par l'activité mentale nécessaire à la compréhension des récits se déclarent totalement désintéressés par les approches expérimentales » (ibid. : 6).

Les arguments présentés par Marie-Laure Ryan sont convaincants, même si j’ai moimême défendu l'apport indéniable des sciences cognitives pour la théorie littéraire (Schmitt, 20 I2). Toutefois, dans ce cas particulier, elle porte son attention non plus sur la poétique cognitive, mais bien sur la narratologie cognitive. Nous reviendrons brièvement sur la distinction entre ces deux domaines, même s'ils sont souvent interchangeables dans l'esprit de ceux qui les utilisent, Marie-Laure Ryan les différencie à juste titre. Ensuite, elle pointe du doigt ensuite une autre limite de la narratologie cognitive :

« En outre, contrairement aux versions expérimentales des sciences de l'esprit, la narratologie cognitive ne veut pas complètement sacrifier l'intérêt pour le texte, même si elle traite souvent les œuvres comme un prétexte ou guide (cf. l'expression de "tutor text" fréquente chez Herman) pour la démonstration d'idées empruntées à la droite » (ibid. : 6-7).

Ces obstacles sont certains et substantiels. Cependant, il me semble que le plus important est l'inaccessibilité des données scientifiques qui, pourtant, sont les seules à pouvoir donner une cohérence à la narratologie cognitive : par exemple, être capable de mesurer l'impact cognitif ou émotionnel chez un lecteur d'un changement de modalité narrative ou de focalisation, du passage soudain d'une narration homodiégétique à une narration hétérodiégétique, ou encore de la lecture d'un même passage narré successivement selon ces deux formes de narration. De telles expériences scientifiques donneraient une réelle cohérence disciplinaire à la narratologie cognitive :le sujet d'étude demeurerait indubitablement dans le cadre de la narratologie et l'aspect scientifique permettrait de pousser le plus loin possible ce que j'ai appelé le pôle de la réception sur l'axe du domaine narratologique, tout en conservant l'intégrité de cet axe.

En effet, à l'instar de Marie-Laure Ryan (2015:6), je pense que l'importation des sciences cognitives dans la théorie littéraire relève du « bricolage interdisciplinaire 
qui emprunte des idées à droite et à gauche $\rangle^{10}$. Mais, contrairement à elle, je reste persuadé que cela ne représente pas un problème en soi ; ou du moins, que le risque mérite d'être pris. Le «bricolage interdisciplinaire » peut quelquefois nous apporter des connaissances que la cohérence disciplinaire n'est plus en mesure de fournir. II faut juste accepter que les conclusions potentiellement issues de la poétique cognitive demeurent, dans un premier temps en tout cas, relatives ou plus fragiles que l'étude des différents modes de narration menée par Gérard Genette, par exemple ; mais les enjeux et les objectifs ne sont de toute façon pas les mêmes. II est simplement important de renouveler le champ des études littéraires, même si cela doit se faire en « bricolant ».

Néanmoins, de nombreux aspects de la poétique cognitive ou de ce qu'on appelle aussi la théorie du récit ne relèvent pas selon moi de la narratologie - et sur ce point je diffère de l'analyse de Raphaël Baroni qui emploie narratologie et théorie du récit de manière interchangeable - même si certains cas restent équivoques. C'est, par exemple, le cas de l'étude des phénomènes de visualisation lors de la lecture, un champ primordial (mais en aucun cas narratologique) trop longtemps négligé par les études littéraires, qui influe sur, voire interfère avec, le sens et le contenu d'un texte. II en va de même pour les phénomènes d'attention et d'inattention, de zones de haute et basse intensité dans un récit, mais aussi, pour citer Raphaël Baroni (2016:221) à nouveau, pour « les usages de la fiction, l'expérience immersive dans le monde raconté, l'empathie envers les personnages, l'évaluation éthique de leurs actions, etc. ». De plus, je demeure sceptique sur les éventuelles applications narratologiques de la très large notion de typification du récit, inspirée des travaux d'Alfred Schutz (1962), Roger Schank et Robert Abelson (1977) (et leur schema et scripts) ou encore de George Lakoff (1987) (et ses « Idealised Cognitive Models ») pour ne citer que quelques exemples; sachant que cette typification est bien souvent culturelle et dépasse le cadre étroit du récit que nous lisons. De nombreux autres aspects de la poétique cognitive (l'étude des émotions suscitées par les structures diégétiques, pour n'en citer qu'un) confirment cette logique centrifuge du champ. Toutefois, ce qui fait la spécificité incontournable, le noyau irréductible de la narratologie, réside justement dans sa logique centripète, son cadre étroit que je définirais par ce qu'on appelle en anglais le close reading, une lecture attentive du texte. La narratologie demeure « prisonnière » d'une étude étroite du texte. C'est à la fois sa force et ses limites, logique que James Phelan et Peter J. Rabinowitz ont parfaitement appréhendée lorsqu'ils ont décidé d'adopter comme méthodologie d'analyse ce qu'ils ont appelé Theory Practice, dont James Phelan (1996:89) donne une définition éloquente: « C'est-à-dire, plutôt que d'invoquer la théorie rhétorique pour "résoudre" les problèmes interprétatifs posés par l'histoire, j'aimerais que la théorie elle-même puisse être modifiée ou compliquée par la nouvelle d'Hemingway [que James

\footnotetext{
${ }^{10}$ M.-L. Ryan semble ici revenir avec ironie sur ce que David Herman (2003:5) a appelé le « tournant interdisciplinaire des études sur la narrativité » (« the cross-disciplinary narrative turn »).
} 
Phelan étudie dans ce chapitre ]" ». La lecture attentive d'un passage n'est plus là uniquement pour illustrer un aspect théorique, mais pour éventuellement modifier la théorie dont on se sert pour la mener; il s'agit bien d'une influence mutuelle. À une époque où l'identité de la narratologie était directement menacée par l'essor des pratiques transdisciplinaires avec de surcroit une amplitude croissante de la théorie du récit, James Phelan et Peter J. Rabinowitz ont ainsi renforcé la spécificité même de la pratique narratologique, tout en l'autorisant à ne pas se cantonner à l'étude formaliste en lui octroyant notamment une dimension rhétorique.

Le socle de la narratologie réside donc autant sinon plus dans sa pratique que dans sa théorie. Elle doit savoir « se contenter » de l'étude de textes spécifiques; ou, pour dire les choses autrement, elle se doit d'être spécifique. Cela peut quelquefois en limiter l'intérêt, en particulier lorsque, lors d'un colloque, on assiste à la présentation de l'étude d'un texte que l'on a pas lu et dont on ne maitrise pas les enjeux, ou dont les caractéristiques narratives ne sont pas exportables au champ littéraire sur lequel on travaille. Certes, on peut admirer le brio d'une analyse, mais si l'on en retire rien pour sa propre recherche, il est parfois difficile de maintenir son attention.

Le champ de la narratologie reste ou se doit de rester étroit car sa pratique l'est, tout simplement. Sa théorie est en quelque sorte conditionnée par la praxis. À une époque où les initiatives transdisciplinaires sont encouragées (et cela me semble un point positif), elle peut apparaître moins attractive tant elle est déterminée, cela dès son origine, par ce qui lui confère sa raison d'être : le texte, au singulier, et ses structures narratives intrinsèques qui ne sont jamais les mêmes d'un texte à l'autre (même s'il est toujours possible de dégager certaines tendances générales, avec la perte de précision que cela implique). La théorie du récit qui, en définitive concerne tous les champs susceptibles d'être convoqués, peut se permettre de prendre le risque de la généralisation, de travailler sur des modèles narratifs larges, ou des schémas cognitifs non spécifiques dans le cas de la poétique cognitive. Prendre occasionnellement des exemples pour illustrer sa démarche analytique diffère de l'analyse étroite décrite ci-dessus, nous savons tous en effet que ce choix n'est pas innocent et que la nature de ces exemples est conditionnée par ce que nous cherchons à dire, et non l'inverse. Cette façon de procéder, forcément plus inclusive, permet à un plus grand nombre de chercheurs de « s'y retrouver », au débat scientifique de ne pas être limité ou entravé par les idiosyncrasies d'une forme narrative en particulier. Theory Practice replace ces idiosyncrasies au centre de la pratique narratologique, posant comme condition à toute élaboration théorique l'observation précise de faits narratifs inhérents à un texte en particulier. En cela, James Phelan et Peter J. Rabinowitz, j'en suis persuadé, ont saisi l'essence de ce qu'est la narratologie classique ou post-classique.

\footnotetext{
" "That is, rather than simply invoke rhetorical theory to "solve" the interpretive problems of the story, I would like the theory itself to be open to revision or complication by Hemingway's story ».
} 
En dernier lieu, les dangers qui menacent la narratologie, évoqués par Raphaël Baroni (2016 : 220) dans la première partie de son article, restent bien réels et peuvent se résumer à l'équation suivante : il est périlleux de résister à « la vague narrativiste », de rester « dans son coin » alors que les analyses narratives sortent des frontières du littéraire pour aller à la rencontre d'autres formes de récit - c'est ce qu'il appelle « l'empire du récit » (ibid. : 22 I) -, mais il est tout aussi dangereux de perdre son objet premier, le texte littéraire (et j'inclus les récits graphiques dans cette catégorie) et le cadre étroit de sa pratique.

\section{La narratologie à l'Université}

Je souhaiterais terminer sur un point plus subjectif, relevant principalement du ressenti et du parcours personnel, qui concerne la place institutionnelle de la narratologie. S'il existe quelques enclaves, principalement internationales (ISSN et ENN - European Narratology Network) mais aussi françaises (L'École des hautes études en sciences sociales et le Laboratoire interdisciplinaire récits, cultures, sociétés à Nice), énumérées par Raphaël Baroni, il est aussi vrai que la narratologie, discipline somme toute assez jeune, donne déjà des signes d'épuisement. A-t-elle pour autant « reflué dans les limbes des modes académiques, tel un territoire abandonné que l'on ne visiterait plus que dans le cadre d'une croisière sur le fleuve de l'histoire des idées » (ibid. : 220) ? C'est probablement vrai pour la France, malgré les quelques poches de résistance susmentionnées, pas forcément pour d'autres pays européens (Allemagne, Danemark, Suisse, par exemple) qui représentent des exceptions, mais des exceptions dynamiques. La narratologie donne aussi l'impression de maintenir sa vitesse de croisière aux États-Unis, une vitesse pas forcément fulgurante mais, encore une fois, constante. Et si cette vitesse vient à décroitre, c'est avant tout pour des raisons contextuelles, liées en grande partie non à un désintérêt pour la narratologie, mais pour la littérature en général. Une fois de plus, il ne faut pas oublier que le destin de la narratologie reste pour l'instant lié à l'objet littéraire, dont l'étude demeure la raison principale pour laquelle elle a vu le jour. Bien sûr, elle peut être utilisée pour les récits visuels et audiovisuels, j'en veux pour preuve l'émergence de la narratologie transmédiale, ce qui peut en garantir la pérennité, mais il est difficile pour l'instant d'imaginer la narratologie sans la littérature.

Les remarques de James Phelan, citées par Raphaël Baroni, me semblent décrire parfaitement la situation en France. Quel que soit le mode d'analyse dont on se réclame (et la narratologie en est un parmi d'autres), l'étude de la littérature se fait principalement au sein des universités, et, plus spécifiquement, des départements de sciences humaines (lettres modernes, classiques, ou départements de langues vivantes). À l'instar de ce qui se produit outre-Atlantique (mais aussi dans une majorité de pays proposant un enseignement universitaire), « ces départements restent liés au paradigme de l'histoire littéraire comme principe fondamental d'organisation de la connaissance » (Phelan, in : Baroni, 2016 : 220). Un chercheur en littérature en France est avant tout défini par une 
aire géographique, puis, en spécificité de second rang, par une période historique. Pour prendre un exemple précis, le mien en l'occurrence, je suis chercheur en littérature américaine contemporaine : la spécificité de premier rang étant la littérature américaine, celle de second rang la littérature américaine contemporaine. Cette classification laisse entendre qu'il y a forcément plus de liens entre deux auteurs américains, même si l'un provient de la côte ouest et l'autre de la côte est, ou même s'ils ont une différence d'âge de deux ou trois décennies, qu'entre un auteur britannique et un auteur américain appartenant à la même génération et étant rattachés au même mouvement littéraire (John Barth et John Fowles, par exemple, pour le postmodernisme). Selon moi, cela correspond à une logique fortement déficiente, mais c'est pourtant celle-ci qui régit les modes de recrutement des enseignants-chercheurs en littérature dans notre pays ; nous retrouvons ici l'identité « monologique » évoquée par Raphaël Baroni (ibid. : 229). En outre, lorsqu'on est défini par une zone géographique de par son recrutement sur un poste au profil spécifique, il demeure très compliqué d'en sortir. Le contexte actuel - diminution du nombre de postes d'enseignants-chercheurs combinée à un désintérêt grandissant pour la littérature - n'est pas propice à une plus grande fluidité dans la définition de nos profils et de nos compétences. La narratologie semble bien secondaire parmi toutes ces préoccupations et se définir comme narratologue, comme le fait remarquer James Phelan à la suite des propos cités ci-dessus, relève du luxe, ou, selon moi, de l'anecdotique dans le déroulement d'une carrière dans les humanités. Le fait que l'étude de la littérature en France au début du XXle siècle soit encore régie par ces considérations géographiques et non par les moyens utilisés pour mener à bien cette étude peut inciter à un certain pessimisme quant au sort de la narratologie.

Je n'ai d'ailleurs personnellement jamais eu le sentiment que la narratologie ait connu un âge d'or, même s'il est vrai que j'ai commencé à étudier la littérature à un moment où elle était censée entamer son déclin (début des années 90). Mais à l'exception de lieux d'excellence comme le Collège de France ou l'École des hautes études en sciences sociales qui n'ont pas à obéir à la logique segmentaire du Conseil national des universités ( $\mathrm{CNU}$ ) et, en conséquence, bénéficient d'une grande liberté théorique, il est rare en France de participer à un colloque sur la littérature - et ce quel que soit le thème affiché -, et d'avoir l'opportunité d'écouter une analyse littéraire structurée par une logique narratologique. II me semble difficile de contester le fait que la principale stratégie analytique en France en ce qui concerne les études littéraires demeure l'approche symbolico-thématique, avec parfois pour les œuvres les plus anciennes des considérations contextualisantes, d'ordre historique. II y a quelques années, j'ai moi-même co-organisé un colloque portant sur le thème des narrateurs fous, thème éminemment narratologique s'il en est un, le texte de cadrage abordant sans équivoque le problème maintes fois traités de la fiabilité de l'instance narrative ; pourtant, de manière surprenante, très peu de participants ont choisi l'angle narratologique pour traiter le sujet. Le mode opératoire de ce genre de manifestations scientifiques demeure peu ou prou le même depuis le début de ma carrière universitaire : appliquer le thème du colloque à sa propre zone de recherche, zone géographique comme nous l'avons vu, ou se résumant à un auteur ou un mouvement littéraire. 


\section{Conclusion}

Se déclarer comme narratologue est une démarche quelque peu désespérée lorsqu'il s'agit de sa visibilité professionnelle ou, de manière plus pragmatique, d'obtenir un poste d'enseignant-chercheur. II faut donc avant tout être spécialiste d'un auteur ou d'une période bien définie de l'histoire littéraire d'un pays. Cela m'a toujours paru singulier car cette spécialisation n'est en aucun cas garante d'une connaissance approfondie des mécanismes narratologiques qui gouvernent une œuvre et le sens, les affects et les percepts qu'elle génère ; selon moi, même une approche thématique devrait emprunter a minima ce détour narratologique. Bien sûr, de nombreux collègues sont en désaccord avec cette assertion. Cette désapprobation est-elle croissante ? I| est difficile de répondre à cette question, mais une chose apparait certaine : rares sont les approches narratologiques en dehors de lieux et publications déjà évoqués, alors que les colloques et journée d'études consacrés à la littérature, ou plus généralement à la fiction, sont pléthore. De plus, le désintérêt relatif que connait la littérature, notamment chez les jeunes générations, peut créer des crispations au sein de la communauté des chercheurs en littérature, les poussant à simplifier leurs stratégies d'analyse de textes, et à se concentrer sur ce qui peut être perçu comme l'essentiel : les programmes des concours de l'enseignement secondaire, et donc, principalement, une approche historico-thématique de la littérature (avec bien évidemment, quelques exceptions).

Comment garantir alors une place à la narratologie dans le paysage universitaire français des sciences humaines? Les solutions préconisées par Raphaël Baroni sont incontournables : création d'une section CNU consacrée à la théorie littéraire ou, à défaut, des postes d'enseignants-chercheurs profilés en théorie littéraire (dans le cadre des sections existantes) et introduction de problématiques narratologiques dans les programmes des concours. En l'absence de telles mesures, on peut en effet être pessimiste quant à l'avenir de cette discipline qui continue de donner, non pas une raison d'être mais une raison d'analyser à certains chercheurs en France, et à de nombreux chercheurs à travers le monde. Indéniablement, le destin de la narratologie reste lié à celui des politiques universitaires, et cela représente sans doute sa limite principale. On pourrait rétorquer que cette limite est inhérente à grand nombre de matières, disciplines ou encore théories enseignées à l'université ; je n'en suis pourtant pas certain. S'il est vrai que les sciences dites (de manière sommaire) « dures » nécessitent la maîtrise d'un langage qui est profondément différent de celui de la doxa, et qui ne peut atteindre cette dernière que sous forme de vulgarisation ou de simplifications radicales, les sciences humaines ont réussi à maintenir un contact plus ou moins étroit avec un public non universitaire sans forcément consentir à faire des concessions infamantes, pour preuve le succès occasionnel (et toujours relatif bien évidemment) d'un ouvrage d'histoire ou de sociologie, d'un essai littéraire ou philosophique, résumant un travail de recherche dont les angles ont été arrondis pour une compréhension facilitée. Les sciences humaines, même si leur statut scientifique est loin d'être admis par tous, conservent ce privilège de pouvoir dialoguer, dans une langue commune, avec 
la doxa et d'être en position d'exporter certaines de ses idées. Or, il s'avère que parmi toutes les disciplines relevant des humanités, la narratologie appartient à la catégorie présentant un potentiel faible d'exportabilité, notamment pour les raisons suivantes : en général, elle n’a pas pour objectif de réfléchir à la société dans laquelle nous vivons et son discours sur les faits littéraires n'est pas thématique (donc généralisable) ; elle présente un lexique plus technique que d'autres approches d'analyse littéraire ; enfin, plus que produire des idées, elle élabore des concepts dont la finalité est avant tout pragmatique et ne s'applique qu'à l'étude des textes littéraires (et maintenant donc, à la fiction au sens large). En d'autres termes, la narratologie m'apparait avant tout comme une pratique (universitaire) et, pour toutes les raisons évoquées plus haut, mais aussi dans l'article de Raphaël Baroni, une pratique qui tend à s'essouffler. Son périmètre est circonscrit par le paysage universitaire actuel et celui-ci, en tout cas en France, ne semblant pas lui être particulièrement favorable, nous pouvons exprimer une certaine inquiétude sur son avenir.

\section{Références}

Alber J., Fludernik M., eds, 2010 , Postclassical Narratology:Approaches and Analyses, Columbus, Ohio State University Press.

Baroni R., 2016, « L'empire de la narratologie, ses défis et ses faiblesses », Questions de communication, 30, pp. 219-238.

Bloom P., 20 I2, «Who Cares about the Evolution of Stories? », Critical Inquiry, 38, 2, pp. 388-393.

Booth W., 1961, The Rhetoric of Fiction, Chicago, Chicago University Press.

Genette G., 1994, L'CEuvre de l'art : immanence et transcendance, Paris, Éd. Le Seuil.

Genette G., 1997, L'Guvre de l'art : la relation esthétique, Paris, Éd. Le Seuil.

Herman D., 2003, Narrative Theory and the Cognitive Sciences, Stanford, Center for the Study of Language and Information.

Kramnick J., 20 I I, « Against Literary Darwinism », Critical Inquiry, 37, pp. 31 5-347.

Lakoff G., 1987, Women, Fire, and Dangerous Things, Chicago, University of Chicago Press.

Meister J. C., 2013, « Narratology », The Living Handbook of Narratology. Accès : http://wikis. sub.uni-hamburg.de/lhn/index.php/Narratology.

O'Kelly D., 20 I2, « Du temps perdu au temps retrouvé : Proust face à Genette », Modèles linguistiques, 65. Accès : http://ml.revues.org/245.

Passalacqua F., Pianzola F., 20 I 6, « Epistemological Problems in Narrative Theory: Objectvist vs. Constructvist Paradigm », pp. 195-2 17, in: Baroni, R., Revaz, F., eds, Narrative Sequence in Contemporary Narratology, Columbus, Ohio State University Press.

Phelan J., 1996, Narrative as Rhetoric. Technique, Audiences, Ethics, Ideology, Columbus, Ohio State University Press. 
Pinker S., 1994, The Language Instinct.The New Science of Language and Mind, Harmondsworth, Penguin.

Rabinowitz P. J., 1987, Before Reading. Narrative conventions and the politics of interpretation, Ithaca, Cornell University Press.

Ryan M.-L., 2015, « Narratologie et sciences cognitives : une relation problématique », Cahiers de narratologie, 28. Accès : http://narratologie.revues.org/7171.

Schank R., Abelson R., 1977, Scripts, Plans, Goals, and Understanding. An Inquiry into Human Knowledge Structures, Hillsdale, N. J., Lawrence Erlbaum Associates.

Schmitt A., 2012, « De la poétique cognitive et de ses (possibles) usages », Poétique, I70, pp. $\mid 43-162$.

Schutz A., 1962, « Common-Sense and the Scientific Interpretation of Human Action », in: Natanson M., ed., Collected Papers, The Hague, Nijhoff.

Segal E., 20I5, « L'école de Tel Aviv, une approche rhétorique et fonctionnaliste du récit », Cahiers de narratologie, 28. Accès : http://narratologie.revues.org/7289.

Strawson G., 2004, « Against Narrativity », Ratio, 17, pp. 428-52. 